Tropical Journal of Pharmaceutical Research November 2015; 14 (11): 2047-2053

ISSN: $1596-5996$ (print); 1596-9827 (electronic)

(c) Pharmacotherapy Group, Faculty of Pharmacy, University of Benin, Benin City, 300001 Nigeria.

All rights reserved.

Available online at http://www.tjpr.org

Original Research Article

http://dx.doi.org/10.4314/tjpr.v14i11.14

\title{
Synthesis and Evaluation of some New 5-Substituted-1,3,4- oxadiazol-2yl-4-(morpholin-4yl Sulfonyl)benzyl Sulfides as Antibacterial Agent
}

\author{
Aziz-ur-Rehman ${ }^{1 *}$, S Gul ${ }^{1}$, MA Abbasi ${ }^{1}, \mathrm{~K}_{\text {Nafeesa }}{ }^{1}, \mathrm{MN}$ Akhtar ${ }^{4}, \mathrm{KM} \mathrm{Khan}{ }^{2}$, I \\ Ahmad $^{3}$ and S Afzal ${ }^{3}$ \\ ${ }^{1}$ Department of Chemistry, Government College University, Lahore-54000, ${ }^{2} \mathrm{HEJ}$ Research Institute of Chemistry, International \\ Center for Chemical and Biological Sciences, University of Karachi, Karachi-75270, ${ }^{3}$ Department of Pharmacy, The Islamia \\ University of Bahawalpur, Bahawalpur-63100, Pakistan, ${ }^{4}$ Faculty of Industrial Sciences \& Technology, University of Malaysia \\ Pahang (UMP), Lebuhraya Tun Razak 26300 Gambang, Kuantan, Malaysia
}

*For correspondence: Email: azizryk@yahoo.com; Tel: (+92)-42-111000010 Ext.450

Received: 18 November 2014

Revised accepted: 23 September 2015

\begin{abstract}
Purpose: To synthesise a new series of 5-substituted-1,3,4-Oxadiazol-2yl-4-(morpholin-4yl sulfonyl)benzyl sulfide and evaluate their antibacterial activity.

Methods: Different organic acids were converted consecutively into corresponding esters, hydrazides and 5-substituted-1,3,4-Oxadiazol-2-thiols (4a-e). The targets, 6a-e were synthesized by stirring 4a-e with 4-(4-(bromomethyl)phenylsulfonyl) morpholine (5) in the presence of N,N-dimethylformamide (DMF) and sodium hydride $(\mathrm{NaH})$. All the structures were elucidated by modern spectroscopic techniques and screened against bacteria using standard procedure and ciprofloxacin drug as positive control.

Results: The yield of the synthesized compounds (4a-e and 6a-e) were moderate (65 - $90 \%$ ). Compounds 6a-e had antibacterial activity against Pseudomonas aeruginosa, Bacillis subtilis and Staphylococcus aureus while some had activity against the other bacteria used. One of the compounds, $6 \boldsymbol{b}$, exhibited significant activity against all the bacterial strains, i.e., S. typhi (-), E. coli (-), K. pneumoniae (-), $P$. aeruginosa (-), B. subtilis (+) and $S$. aureus (+) with MIC ( $\mu M$ ) values of $11.01 \pm$ $0.31,15.37 \pm 3.33,16.11 \pm 1.14,9.70 \pm 1.96,10.01 \pm 2.70$ and $9.15 \pm 0.29$, respectively. However, none of the compounds had any inhibitory activity against any bacteria as high as that of ciprofloxacin. Conclusion: Five new compounds with antibacterial activities have been synthesized. Their potential as therapeutic agents is, however, yet to be evaluated.
\end{abstract}

Keywords: 1,3,4-Oxadiazole, Benzyl sulfide, 4-(4-(bromomethyl)phenylsulfonyl)morpholine, Spectral analysis, Antibacterial activity

Tropical Journal of Pharmaceutical Research is indexed by Science Citation Index (SciSearch), Scopus, International Pharmaceutical Abstract, Chemical Abstracts, Embase, Index Copernicus, EBSCO, African Index Medicus, JournalSeek, Journal Citation Reports/Science Edition, Directory of Open Access Journals (DOAJ), African Journal Online, Bioline International, Open-J-Gate and Pharmacy Abstracts

\section{INTRODUCTION}

Organic chemists are interested in designing and synthesizing fresh and therapeutically active compounds, useful to alleviate different disorders and diseases. Scientists are facing a serious problem of increased resistance of microbes against the traditional antimicrobial drugs and so are interested in developing new biologically active compounds with excellent therapeutic activity. Oxadiazole is a class of heterocyclic organic compounds that is known because of its magnificent therapeutic potential. Thousands of oxadiazole and their derivatives have been 
synthesized and evaluated for different antimicrobial and enzyme inhibition activities. Oxadiazole possesses splendid antiinflammatory [1,2], fungicidal [3], insecticidal [4], herbicidal [5], antibacterial [6], antitumor [7], antitubercular [8], antiviral, anticonvulsant and analgesic activities [9]. Furthermore, morpholine and its analogues are important in pharmaceutics due to their great enzyme inhibition potential, antimicrobial and antioxidant activity $[10,11]$.

Synthesis of new morpholine containing compounds is the area of interest for the pharmacists because therapeutically potent compounds are needed to cure different malfunctions and diseases [12]. This current work (an extension of previous work, [13-15]) aimed to synthesize poly-functional compounds having 1,3,4-oxadiaozloe and sulfa morpholine moiety together in an individual molecule. It was expected that 2,5-disubstituted 1,3,4-oxadiazole ring along with the potential morpholine moiety will boost up the antibacterial activity of the molecule and hence the synthesized compounds were screened against clinically isolated bacteria.

\section{EXPERIMENTAL}

All the chemicals and analytical grade solvents were purchased through local suppliers. Melting points were taken on Griffin and George melting point apparatus by open capillary tube method. TLC plate $\mathrm{F}_{256} 20 \times 20 \mathrm{~cm}$ coated with silica gel was used to detect the purity of synthesized compounds, developed by different ratios of $n$ Hexane and EtOAc as solvent system. With the help of $\mathrm{KBr}$ pellet method, IR spectrum was recorded by using Jasco-320-A spectrophotometer. ${ }^{1} \mathrm{H}-\mathrm{NMR}$ spectra were recorded on Bruker spectrometers at frequency of $400 \mathrm{MHz}$, taken in deuterated chloroform, indicating chemical shifts value in ppm taking TMS as reference standard.

\section{Synthesis of ethyl esters of different substituted carboxylic acids (2a-e)}

The organic acids [(5 g, Piperonylic acid (1a); 3,4-(Methylenedioxy)cinnamic acid (1b); Nicotinic acid (1c); 2-naphthyl acetic acid (1d); 3,5-dinitro2-methyl benzoic acid (1e)] were taken in 250 $\mathrm{mL}$ round bottom flask and dissolved in $20 \mathrm{~mL}$ ethanol along with $2.5 \mathrm{~mL}$ concentrated $\mathrm{H}_{2} \mathrm{SO}_{4}$. The mixture was refluxed for 2-3 h. TLC was used to monitor the reaction completion by using $\mathrm{n}$-hexane and EtOAc as solvent system. On completion, reaction contents were shifted to a separating funnel containing distilled $\mathrm{H}_{2} \mathrm{O}$ (20 $\mathrm{mL}$ ). The contents were neutralized using concentrated $\mathrm{Na}_{2} \mathrm{CO}_{3}$ solution (15\%). Diethyl ether was added to the separating funnel followed by shaking, allowed to separate into two layers and the ethyl esters (2a-e) were recovered following by evaporating the diethyl ether.

\section{Synthesis of different hydrazides (3a-e)}

The ethyl esters (25 mmol, 3a-e) were taken in $250 \mathrm{~mL}$ RB flask and dissolved in methanol (60 $\mathrm{mL})$. Hydrazine hydrate $(80 \%, 0.05-0.07 \mathrm{~mol})$ was introduced to the reaction flask along with stirring for 4-5 $\mathrm{h}$ at room temperature. Some of the hydrazides were formed at room temperature while some esters got altered on refluxing with constant stirring. Completion of reaction was monitored by TLC using $n$-hexane and EtOAc as solvent system. On completion, cold distilled $\mathrm{H}_{2} \mathrm{O}$ was added to obtain the precipitates. Precipitates of hydrazides (3a-e) were filtered, washed with $\mathrm{H}_{2} \mathrm{O}$ and dried.

\section{Synthesis of 5-substituted-1,3,4-oxadiazole-2- thiols (4a-e)}

The hydrazides $(0.01 \mathrm{~mol}$, 3a-e) were taken in $250 \mathrm{~mL}$ RB flask, dissolved in ethanol and $\mathrm{CS}_{2}$ $(0.01 \mathrm{~mol})$ was added followed by the addition of $\mathrm{KOH}(0.03 \mathrm{~mol})$ to provide basic media. The mixture was refluxed for $5-6 \mathrm{~h}$ and reaction progress was monitored by TLC. The reaction completion was confirmed by TLC and the reaction mixture was acidified $(\mathrm{pH}=2)$ to remove un-reacted hydrazides in the form of salts and also to convert thiol group of oxadiazole into acidic form. Precipitates (4a-d) were filtered, washed with $\mathrm{H}_{2} \mathrm{O}$ and dried. Precipitates were recrystallized by methanol.

\section{Synthesis of 4-(4-(bromomethyl)phenyl sulfonyl)morpholine (5)}

Morpholine $(0.005 \mathrm{~mol})$ was added into a round bottom flask containing basic aqueous solution with a pH 9-10. Equimolar 4-(bromomethyl) benzenesulfonyl chloride was introduced to the reaction flask with constant stirring under dynamic $\mathrm{pH}$ control. The completion of reaction was monitored via TLC utilizing different ratios of $\mathrm{n}$-hexane and EtOAc. On completion, reaction mixture was acidified $(\mathrm{pH}=2)$ to obtain the product (5). Precipitates were filtered, washed with water and dried.

\section{Synthesis of 5-substitued-1,3,4-Oxadiazole-2- yl 4-(morpholin-4yl sulfonyl)benzyl sulfide (6a-e)}

5-substituted-1,3,4-oxadiazole-2-thiols $(0.1 \mathrm{~g}, 4 \mathrm{4a}-$ e) were taken in $50 \mathrm{~mL}$ RB flask and dissolved in 
dimethylformamide. On complete dissolution, 2 $\mathrm{mg} \mathrm{NaH}$ was added and stirred for half an hour. The electrophile, 4-(4-(bromomethyl)phenyl sulfonyl) morpholine (5) was added to the reaction mixture, and stirring was continued for further 1-2 h. TLC was used to check the reaction completion by using $\mathrm{n}$-hexane and EtOAc as a solvent system. On completion, cold distilled water was added to get the precipitates which were then filtered, washed with $\mathrm{H}_{2} \mathrm{O}$ and dried. The pathway for the synthesis of 6a-e is provided in scheme 1 while the different 5substituted aryl/aralky groups of synthesized compounds are shown in Table 1.
In summary, the synthesis was carried out in different steps. First, the different organic acids (1a-e) were converted into subsequent ethyl esters (2a-e) by refluxing in concentrated $\mathrm{H}_{2} \mathrm{SO}_{4}$ and ethanol for 2-3 $\mathrm{h}$. Second, the ethyl esters (2a-e) were consequently transformed into hydrazides (3a-e) by refluxing with hydrazine hydrate $(80 \%)$ using ethanol as a solvent for $4-5$ h. Third, the hydrazides (3a-e) were cyclized to 5-substituted-1,3,4-oxadiazoles-2-thiols (4a-e) by refluxing for 5-6 $\mathrm{h}$ with $\mathrm{CS}_{2}$ in the presence of $\mathrm{KOH}$. The products were acquired by acidifying the reaction mixture. The electrophile 5 was synthesized by the reaction of morpholine with 4-<smiles>[R]C(=O)NNN</smiles>

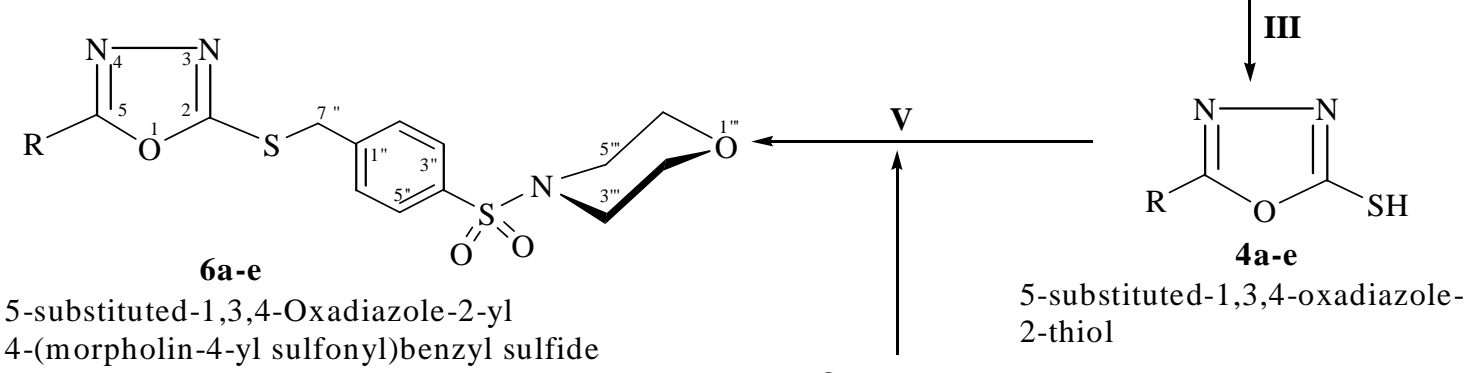
4-(morpholin-4-yl sulfonyl)benzyl sulfide<smiles>CC1CNCCCN1S(=O)(=O)c1ccc(CBr)cc1</smiles>

Scheme-1: Outline for the synthesis of 5-substituted-1,3,4-Oxadiazole-2-yl 4-(morpholin-4-ylsulfonyl)benzyl sulfide. Reagents \& conditions: (I) $\mathrm{H}_{2} \mathrm{SO}_{4} / \mathrm{EtOH} /$ refluxing for $2-3 \mathrm{~h}$ (II) $\mathrm{N}_{2} \mathrm{H}_{4} / \mathrm{MeOH} /$ stirring for $4-5 \mathrm{~h}$ (III) $\mathrm{CS}_{2} / \mathrm{KOH} / \mathrm{EtOH} /$ refluxing for 5-6 h (IV) 4-bromomethylbenzenesulfonyl chloride/ $\mathrm{H}_{2} \mathrm{O} / 5 \% \mathrm{Na}_{2} \mathrm{CO}_{3}$ soln./stirring for $1 \mathrm{~h}(\mathrm{~V}) \mathrm{DMF} / \mathrm{NaH} /$ stirring for 2-3 $\mathrm{h}$

Table-1: Different 5-substituted aryl/aralkyl groups of synthesized compounds

Compound


bromomethylbenzenesulfonyl chloride in the presence basic aqueous media. Fourth, these 5substituted-1,3,4-Oxadiazoles-2-thiols (4a-e) were treated with 4-(4-(bromomethyl) phenylsulfonyl)morpholine (5) in the presence of dimethylformamide as solvent and sodium hydride as base. The final products (6a-e) were collected by the addition of cold water. The structures of all the synthesized compounds were established by ${ }^{1} \mathrm{H}-\mathrm{NMR}, \mathrm{IR}$ and mass spectral data as illustrated in experimental section.

\section{Antibacterial activity assay}

The antibacterial activity assay was processed on sterile 96-wells microplates under sterile conditions. Synthesized compounds were screened against four Gram-negative and two Gram-positive bacteria as previously reported [13-15]. Briefly, the test organisms were maintained on stock culture agar medium. Each test sample (in suitable solvent and appropriate dilution) was pipetted into each well $(20 \mu \mathrm{g} / \mathrm{well})$. Freshly maintained overnight bacterial culture $(180 \mu \mathrm{L})$, after dilution with fresh nutrient broth, was poured into each well which was then incubated at $37{ }^{\circ} \mathrm{C}$ for $16-24 \mathrm{~h}$ under cover of a microplate. Absorbance was measured at 540 $\mathrm{nm}$ using microplate reader, before and after incubation and the difference was noted as an index of bacterial growth using ciprofloxacin as reference standard. Percentage inhibition of each bacteria was calculated and the minimum inhibitory concentration (MIC) was measured using suitable dilutions $(5-30 \mu \mathrm{g} /$ well) of each compound.

\section{Statistical analysis}

All the calculations for MIC values were made after performing the experiments in triplicate. The triplicate calculated values were analyzed statistically through Microsoft Excel 2010 with CL of $85 \%$. The results are offered as mean \pm SEM.

\section{RESULTS}

\section{Spectral characteristics of the synthesized compounds}

\section{5-(1,3-Benzodioxol-5-yl)-1,3,4-oxadiazol-2yl-4- (morpholin-4yl sulfonyl)benzyl sulfide (6a)}

Light pink amorphous solid; Yield: $88 \%$; M.P: 140-142 ${ }^{\circ} \mathrm{C}$; molecular formula: $\mathrm{C}_{20} \mathrm{H}_{19} \mathrm{~N}_{3} \mathrm{O}_{6} \mathrm{~S}_{2}$; Mol. Wt: 461; IR $\left(\mathrm{KBr}, \mathrm{cm}^{-1}\right) v_{\max }: 3439$ (C-H stretching of benzene ring), 2929 (C-H stretching $\left.-\mathrm{CH}_{2}\right), \quad 1623$ \& $1610 \quad(\mathrm{C}=\mathrm{N}$ stretching of Oxadiazole ring), 1463 ( $\mathrm{Ar}-\mathrm{C}=\mathrm{C}$ aromatic stretching band), 1408 (- $\mathrm{SO}_{2}$ - stretching), 1331 \& 1143 (C-O-C bond stretching); ${ }^{1} \mathrm{H}-\mathrm{NMR}$ (400 $\left.\mathrm{MHz}, \mathrm{CDCl}_{3}, \delta / \mathrm{ppm}\right) ; 7.56(\mathrm{~d}, J=8.0 \mathrm{~Hz}, 2 \mathrm{H}, \mathrm{H}-$ 2" \& H-6"), 7.53 (d, J = 8.4 Hz, 2H, H-3" \& H-5"), $7.34\left(\mathrm{~d}, J=8.0 \mathrm{~Hz}, 1 \mathrm{H}, \mathrm{H}-6^{\prime}\right), 7.24(\mathrm{~d}, J=2.0 \mathrm{~Hz}$, $\left.1 \mathrm{H}, \mathrm{H}-4^{\prime}\right), 6.76\left(\mathrm{~d}, J=8.4 \mathrm{~Hz}, 1 \mathrm{H}, \mathrm{H}-7^{\prime}\right), 5.89$ (s, $\left.2 \mathrm{H}, \mathrm{CH}_{2}-2^{\prime}\right), 4.38\left(\mathrm{~s}, 2 \mathrm{H}, \mathrm{CH}_{2}-7{ }^{\prime \prime}\right), 3.57$ (t, $J=4.4$ $\mathrm{Hz}, 4 \mathrm{H}, \mathrm{CH}_{2}-2$ "'\& $\mathrm{CH}_{2}-6$ "'), 2.81 (t, $J=4.4 \mathrm{~Hz}$, $\left.4 \mathrm{H}, \mathrm{CH}_{2}-3^{\prime \prime \prime} \& \mathrm{CH}_{2}-5^{\prime \prime \prime)}\right)$ EIMS (m/z): $461[\mathrm{M}]^{+}, 311$ $\left[\mathrm{M}-\mathrm{C}_{4} \mathrm{H}_{8} \mathrm{NO}_{3} \mathrm{~S}\right]^{+}, \quad 271\left[\mathrm{C}_{11} \mathrm{H}_{13} \mathrm{NO}_{3} \mathrm{~S}\right]^{+}, \quad 240$ $\left[\mathrm{C}_{11} \mathrm{H}_{14} \mathrm{NO}_{3} \mathrm{~S}\right]^{+}, 221\left[\mathrm{M}-\mathrm{C}_{11} \mathrm{H}_{14} \mathrm{NO}_{3} \mathrm{~S}\right]^{+}, 189[\mathrm{M}-$ $\left.\mathrm{C}_{11} \mathrm{H}_{14} \mathrm{NO}_{3} \mathrm{~S}\right]^{+}, 149\left[\mathrm{M}-\mathrm{C}_{12} \mathrm{H}_{14} \mathrm{~N}_{3} \mathrm{O}_{3} \mathrm{~S}_{2}\right]^{+}, 147[\mathrm{M}-$ $\left.\mathrm{C}_{12} \mathrm{H}_{14} \mathrm{~N}_{2} \mathrm{O}_{4} \mathrm{~S}_{2}\right]^{+}, \quad 121 \quad\left[\mathrm{M}-\mathrm{C}_{13} \mathrm{H}_{14} \mathrm{~N}_{3} \mathrm{O}_{4} \mathrm{~S}_{2}\right]^{+}, \quad 86$ $\left[\mathrm{C}_{4} \mathrm{H}_{8} \mathrm{NO}\right]^{+}$.

\section{5-[2-(1,3-Benzodioxol-5-yl)ethenyl]-1,3,4- oxadiazol-2yl-4-(morpholin-4yl sulfonyl)benzyl sulfide (6b)}

Light green amorphous solid; Yield: $66 \%$; M.P: 156-158 ${ }^{\circ} \mathrm{C}$; molecular formula: $\mathrm{C}_{22} \mathrm{H}_{21} \mathrm{~N}_{3} \mathrm{O}_{6} \mathrm{~S}_{2}$; Mol. Wt: 487; IR $\left(\mathrm{KBr}, \mathrm{cm}^{-1}\right) v_{\max }: 3438(\mathrm{C}-\mathrm{H}$ stretching of benzene ring), 2927 ( $\mathrm{C}-\mathrm{H}$ stretching $\left.-\mathrm{CH}_{2}\right), 1622$ \& $1611 \quad(\mathrm{C}=\mathrm{N}$ stretching of Oxadiazole ring), 1462 (Ar-C=C aromatic stretching band), 1407 (-SO $\mathrm{SO}_{2}$ - stretching), 1330 \& 1142 (C-O-C bond stretching); ${ }^{1} \mathrm{H}-\mathrm{NMR}$ (300 $\left.\mathrm{MHz}, \mathrm{CDCl}_{3}, \delta / \mathrm{ppm}\right) ; 7.63(\mathrm{~d}, J=8.0 \mathrm{~Hz}, 2 \mathrm{H}, \mathrm{H}-$ 2" \& H-6"), 7.54 (d, J= 8.4 Hz, 2H, H-3" \& H-5"), $7.38\left(\mathrm{~d}, J=8.0 \mathrm{~Hz}, 1 \mathrm{H}, \mathrm{H}-6^{\prime}\right), 7.29(\mathrm{~d}, J=16.5$ $\left.\mathrm{Hz}, 1 \mathrm{H}, \mathrm{H}-9^{\prime}\right), 6.93\left(\mathrm{~d}, J=16.5 \mathrm{~Hz}, 1 \mathrm{H}, \mathrm{H}-8^{\prime}\right)$, $6.88\left(\mathrm{~s}, 1 \mathrm{H}, \mathrm{H}-4^{\prime}\right), 6.70\left(\mathrm{~d}, J=8.4 \mathrm{~Hz}, 1 \mathrm{H}, \mathrm{H}-7^{\prime}\right)$, 5.90 (s, 2H, $\left.\mathrm{CH}_{2}-2^{\prime}\right), 4.43$ (s, 2H, $\left.\mathrm{CH}_{2}-7 "\right), 3.63$ (t, $\left.J=4.2 \mathrm{~Hz}, 4 \mathrm{H}, \mathrm{CH}_{2}-2{ }^{\prime \prime} \& \mathrm{CH}_{2}-6{ }^{\prime \prime}\right), 2.90$ (t, $J=$ 4.2 Hz, 4H, $\left.\mathrm{CH}_{2}-3^{\prime \prime \prime} \& \mathrm{CH}_{2}-5^{\prime \prime \prime}\right)$; EIMS (m/z): 487 $[\mathrm{M}]^{+}, \quad 337\left[\mathrm{M}^{-} \mathrm{C}_{4} \mathrm{H}_{8} \mathrm{NO}_{3} \mathrm{~S}\right]^{+}, \quad 271\left[\mathrm{C}_{11} \mathrm{H}_{13} \mathrm{NO}_{3} \mathrm{~S}\right]^{+}$, $240\left[\mathrm{C}_{11} \mathrm{H}_{14} \mathrm{NO}_{3} \mathrm{~S}\right]^{+}, 247\left[\mathrm{M}-\mathrm{C}_{11} \mathrm{H}_{14} \mathrm{NO}_{3} \mathrm{~S}\right]^{+}, 215$ $\left[\mathrm{M}-\mathrm{C}_{11} \mathrm{H}_{14} \mathrm{NO}_{3} \mathrm{~S}\right]^{+}, 175\left[\mathrm{M}-\mathrm{C}_{12} \mathrm{H}_{14} \mathrm{~N}_{3} \mathrm{O}_{3} \mathrm{~S}_{2}\right]^{+}, 173$ $\left[\mathrm{M}-\mathrm{C}_{12} \mathrm{H}_{14} \mathrm{~N}_{2} \mathrm{O}_{4} \mathrm{~S}_{2}\right]^{+}, \quad 147\left[\mathrm{M}-\mathrm{C}_{13} \mathrm{H}_{14} \mathrm{~N}_{3} \mathrm{O}_{4} \mathrm{~S}_{2}\right]^{+}, 86$ $\left[\mathrm{C}_{4} \mathrm{H}_{8} \mathrm{NO}\right]^{+}$.

\section{5-(3-pyridin)-1,3,4-oxadiazol-2yl-4-(morpholin- 4yl sulfonyl)benzyl sulfide (6c)}

Off white amorphous solid; Yield: $77 \%$; M.P: 161-163 ${ }^{\circ} \mathrm{C}$; molecular formula: $\mathrm{C}_{18} \mathrm{H}_{18} \mathrm{~N}_{4} \mathrm{O}_{4} \mathrm{~S}_{2}$; Mol. Wt: 418; IR $\left(\mathrm{KBr}, \mathrm{cm}^{-1}\right) v_{\max }: 3437(\mathrm{C}-\mathrm{H}$ stretching of benzene ring), 2925 (C-H stretching $\left.-\mathrm{CH}_{2}\right), 1621$ \& $1613 \quad(\mathrm{C}=\mathrm{N}$ stretching of Oxadiazole ring), 1463 ( $\mathrm{Ar}-\mathrm{C}=\mathrm{C}$ aromatic stretching band), 1405 (-SO $\mathrm{SO}_{2}$ - stretching), 1331 \& 1143 (C-O-C bond stretching); ${ }^{1} \mathrm{H}-\mathrm{NMR}$ (400 $\left.\mathrm{MHz}, \mathrm{CDCl}_{3}, \delta / \mathrm{ppm}\right) ; 8.71(\mathrm{~s}, 1 \mathrm{H}, \mathrm{H}-2$ '), 7.91 (br.s, $1 \mathrm{H}, \mathrm{H}-4^{\prime}$ ), $7.75\left(\mathrm{~d}, J=7.6 \mathrm{~Hz}, 1 \mathrm{H}, \mathrm{H}-6^{\prime}\right)$, $7.67(\mathrm{~d}, J=8.0 \mathrm{~Hz}, 2 \mathrm{H}, \mathrm{H}-2 "$ \& H-6"), $7.63(\mathrm{~d}, J=$ $8.8 \mathrm{~Hz}, 2 \mathrm{H}, \mathrm{H}-3 "$ \& H-5"), 7.58 (t, $J=8.4 \mathrm{~Hz}, 1 \mathrm{H}$, $\left.\mathrm{H}-5^{\prime}\right), 4.54$ (s, 2H, $\left.\mathrm{CH}_{2}-7^{\prime \prime}\right) 3.63$ (t, $J=4.4 \mathrm{~Hz}$, $4 \mathrm{H}, \mathrm{CH}_{2}-2$ '"'\& $\mathrm{CH}_{2}-6$ "'), 2.92 (t, $J=4.8 \mathrm{~Hz}, 4 \mathrm{H}$, $\mathrm{CH}_{2}-3^{\prime \prime \prime} \& \mathrm{CH}_{2}-5$ "'); EIMS (m/z): $418[\mathrm{M}]^{+}, 268$ [M$\left.\mathrm{C}_{4} \mathrm{H}_{8} \mathrm{NO}_{3} \mathrm{~S}\right]^{+}, \quad 271\left[\mathrm{C}_{11} \mathrm{H}_{13} \mathrm{NO}_{3} \mathrm{~S}\right]^{+}, \quad 240$ 
$\left[\mathrm{C}_{11} \mathrm{H}_{14} \mathrm{NO}_{3} \mathrm{~S}\right]^{+}, 178\left[\mathrm{M}-\mathrm{C}_{11} \mathrm{H}_{14} \mathrm{NO}_{3} \mathrm{~S}\right]^{+}, 146[\mathrm{M}-$ $\left.\mathrm{C}_{11} \mathrm{H}_{14} \mathrm{NO}_{3} \mathrm{~S}\right]^{+}, 106\left[\mathrm{M}-\mathrm{C}_{12} \mathrm{H}_{14} \mathrm{~N}_{3} \mathrm{O}_{3} \mathrm{~S}_{2}\right]^{+}, 104[\mathrm{M}-$ $\left.\mathrm{C}_{12} \mathrm{H}_{14} \mathrm{~N}_{2} \mathrm{O}_{4} \mathrm{~S}_{2}\right]^{+}, \quad 78 \quad\left[\mathrm{M}-\mathrm{C}_{13} \mathrm{H}_{14} \mathrm{~N}_{3} \mathrm{O}_{4} \mathrm{~S}_{2}\right]^{+}, \quad 86$ $\left[\mathrm{C}_{4} \mathrm{H}_{8} \mathrm{NO}\right]^{+}$.

\section{5-(2-naphthalenylmethyl)-1,3,4-oxadiazol-2yl- 4-(morpholin-4yl sulfonyl)benzyl sulfide (6d)}

Off white amorphous solid; Yield: $64 \%$; M.P: 190-192 ${ }^{\circ} \mathrm{C}$; molecular formula: $\mathrm{C}_{24} \mathrm{H}_{23} \mathrm{~N}_{3} \mathrm{O}_{4} \mathrm{~S}_{2}$; Mol. Wt: 481; IR $\left(\mathrm{KBr}, \mathrm{cm}^{-1}\right) v_{\text {max }}: 3447$ (C-H stretching of benzene ring), 2923 (C-H stretching $\left.-\mathrm{CH}_{2}\right), \quad 1620$ \& $1611 \quad(\mathrm{C}=\mathrm{N}$ stretching of Oxadiazole ring), 1461 (Ar- $\mathrm{C}=\mathrm{C}$ aromatic stretching band), 1404 (-SO ${ }_{2}$ - stretching), 1329 \& 1141 (C-O-C bond stretching); ${ }^{1} \mathrm{H}-\mathrm{NMR}$ (400 $\left.\mathrm{MHz}, \mathrm{CDCl}_{3}, \delta / \mathrm{ppm}\right) ; 8.00(\mathrm{~d}, J=8.0 \mathrm{~Hz}, 1 \mathrm{H}, \mathrm{H}-$ 4'), $7.80(\mathrm{~d}, J=7.6 \mathrm{~Hz}, 1 \mathrm{H}, \mathrm{H}-8$ '), 7.77(d, $J=6.8$ $\left.\mathrm{Hz}, 1 \mathrm{H}, \mathrm{H}-5^{\prime}\right), 7.51\left(\mathrm{~d}, J=8.4 \mathrm{~Hz}, 1 \mathrm{H}, \mathrm{H}^{\prime} 3^{\prime}\right), 7.49$ (s, 1H, H-1'), 7.48-7.44 (m, 2H, H-6' \& $\left.\mathrm{H}-7^{\prime}\right)$, $7.38(\mathrm{~d}, J=8.0 \mathrm{~Hz}, 2 \mathrm{H}, \mathrm{H}-2 "$ \& H-6"), 7.36 (d, $J=$ $8.0 \mathrm{~Hz}, 2 \mathrm{H}, \mathrm{H}-3 "$ \& $\mathrm{H}-5$ "), $4.53\left(\mathrm{~s}, 2 \mathrm{H}, \mathrm{CH}_{2}-2^{\prime}\right)$, $4.29\left(\mathrm{~s}, 2 \mathrm{H}, \mathrm{CH}_{2}-7 "\right), 3.64(\mathrm{t}, \mathrm{J}=4.4 \mathrm{~Hz}, 4 \mathrm{H}$, $\mathrm{CH}_{2}-2$ '"\& $\mathrm{CH}_{2}-6$ '"), 2.87 (t, $\mathrm{J}=4.8 \mathrm{~Hz}, 4 \mathrm{H}, \mathrm{CH}_{2}-$ 3"'\& $\mathrm{CH}_{2}-5$ "'); $\operatorname{EIMS~(m/z):~} 481[\mathrm{M}]^{+}, 331$ [M$\left.\mathrm{C}_{4} \mathrm{H}_{8} \mathrm{NO}_{3} \mathrm{~S}\right]^{+}, \quad 271\left[\mathrm{C}_{11} \mathrm{H}_{13} \mathrm{NO}_{3} \mathrm{~S}\right]^{+}, \quad 240$ $\left[\mathrm{C}_{11} \mathrm{H}_{14} \mathrm{NO}_{3} \mathrm{~S}\right]^{+}, 241\left[\mathrm{M}-\mathrm{C}_{11} \mathrm{H}_{14} \mathrm{NO}_{3} \mathrm{~S}\right]^{+}, 209[\mathrm{M}-$ $\left.\mathrm{C}_{11} \mathrm{H}_{14} \mathrm{NO}_{3} \mathrm{~S}\right]^{+}, 169\left[\mathrm{M}-\mathrm{C}_{12} \mathrm{H}_{14} \mathrm{~N}_{3} \mathrm{O}_{3} \mathrm{~S}_{2}\right]^{+}, 167[\mathrm{M}-$ $\left.\mathrm{C}_{12} \mathrm{H}_{14} \mathrm{~N}_{2} \mathrm{O}_{4} \mathrm{~S}_{2}\right]^{+}, \quad 141 \quad\left[\mathrm{M}-\mathrm{C}_{13} \mathrm{H}_{14} \mathrm{~N}_{3} \mathrm{O}_{4} \mathrm{~S}_{2}\right]^{+}, \quad 86$ $\left[\mathrm{C}_{4} \mathrm{H}_{8} \mathrm{NO}\right]^{+}$.

\section{5-(2-methyl-3,5-dinitrophenyl)-1,3,4-oxadiazol- 2yl-4-(morpholin-4yl sulfonyl)benzyl sulfide (6e)}

Yellow amorphous solid; Yield: 79 \%; M.P: 247$249{ }^{\circ} \mathrm{C}$; molecular formula: $\mathrm{C}_{20} \mathrm{H}_{19} \mathrm{~N}_{5} \mathrm{O}_{8} \mathrm{~S}_{2}$; Mol. Wt: 521; IR $\left(\mathrm{KBr}, \mathrm{cm}^{-1}\right) \quad V_{\max }: 3441$ (C-H stretching of benzene ring), 2933 (C-H stretching $\left.-\mathrm{CH}_{2}\right), \quad 1624$ \& $1607 \quad(\mathrm{C}=\mathrm{N}$ stretching of Oxadiazole ring), 1465 (Ar-C=C aromatic stretching band), 1409 (-SO ${ }^{-}$stretching), 1333 \& 1145 (C-O-C bond stretching); ${ }^{1} \mathrm{H}-\mathrm{NMR}$ (300 $\left.\mathrm{MHz}, \mathrm{CDCl}_{3}, \delta / \mathrm{ppm}\right) ; 7.65(\mathrm{~d}, J=8.1 \mathrm{~Hz}, 2 \mathrm{H}, \mathrm{H}-$ 2" \& H-6"), 7.60 (d, J=8.1 Hz, 2H, H-3" \& H-5"), $7.16\left(\mathrm{~d}, J=2.7 \mathrm{~Hz}, 1 \mathrm{H}, \mathrm{H}-4^{\prime}\right), 7.08(\mathrm{~d}, J=2.7 \mathrm{~Hz}$, $\left.1 \mathrm{H}, \mathrm{H}-6^{\prime}\right), 4.47\left(\mathrm{~s}, 2 \mathrm{H}, \mathrm{CH}_{2}-7^{\prime \prime}\right), 3.65(\mathrm{t}, J=$ $4.5 \mathrm{~Hz}, 4 \mathrm{H}, \mathrm{CH}_{2}-2$ "'\& $\mathrm{CH}_{2}-6$ "'), 2.88 (t, $J=4.8 \mathrm{~Hz}$, $\left.4 \mathrm{H}, \mathrm{CH}_{2}-3^{\prime \prime \prime} \& \mathrm{CH}_{2}-5^{\prime \prime \prime}\right) ; \operatorname{EIMS}(\mathrm{m} / \mathrm{z}): 521[\mathrm{M}]^{+}, 371$
$\left[\mathrm{M}-\mathrm{C}_{4} \mathrm{H}_{8} \mathrm{NO}_{3} \mathrm{~S}\right]^{+}, \quad 271\left[\mathrm{C}_{11} \mathrm{H}_{13} \mathrm{NO}_{3} \mathrm{~S}\right]^{+}, \quad 240$ $\left[\mathrm{C}_{11} \mathrm{H}_{14} \mathrm{NO}_{3} \mathrm{~S}\right]^{+}, 281\left[\mathrm{M}-\mathrm{C}_{11} \mathrm{H}_{14} \mathrm{NO}_{3} \mathrm{~S}\right]^{+}, 249[\mathrm{M}-$ $\left.\mathrm{C}_{11} \mathrm{H}_{14} \mathrm{NO}_{3} \mathrm{~S}\right]^{+}, 209\left[\mathrm{M}-\mathrm{C}_{12} \mathrm{H}_{14} \mathrm{~N}_{3} \mathrm{O}_{3} \mathrm{~S}_{2}\right]^{+}, 207[\mathrm{M}-$ $\left.\mathrm{C}_{12} \mathrm{H}_{14} \mathrm{~N}_{2} \mathrm{O}_{4} \mathrm{~S}_{2}\right]^{+}, \quad 181 \quad\left[\mathrm{M}-\mathrm{C}_{13} \mathrm{H}_{14} \mathrm{~N}_{3} \mathrm{O}_{4} \mathrm{~S}_{2}\right]^{+}, \quad 86$ $\left[\mathrm{C}_{4} \mathrm{H}_{8} \mathrm{NO}\right]^{+}$.

\section{Antibacterial activity}

The percentage inhibition of each bacteria and the MICs are presented in Tables 2 and 3, respectively. All five compounds synthesized had antibacterial activity against $P$. aeruginosa, $B$. subtilis and $S$. aureus while the growth of $S$. typhi, E. coli and K. pneumoniae was inhibited by four, one and two of the compounds, respectively. All the compounds, however, had lower levels of inhibition and higher MIC against the bacteria inhibited than the standard ciprofloxacin.

\section{DISCUSSION}

The intention of the presented research work was to synthesize biologically active molecules. We have synthesized some new 2,5disubstituted 1,3,4-oxadiazole molecules and to find out the anti-bacterial activity of all the synthesized molecules. We synthesized 5substituted-1,3,4-oxadiazol-2yl-4-(morpholin-4yl sulfonyl)benzyl sulfide (6a-e) derivaives in good yields having significant antibacterial activities. Compound 6a was synthesized as a light pink amorphous solid having yield $88 \%$ and melting point $140-142{ }^{\circ} \mathrm{C}$. In the IR spectrum, the characteristic peaks appeared at $3439 \mathrm{~cm}^{-1}$, $1632 \mathrm{~cm}^{-1}, 1443 \mathrm{~cm}^{-1}$ and $1408 \mathrm{~cm}^{-1}$ which were corresponding to $\mathrm{C}-\mathrm{H}$ stretching, $\mathrm{C}=\mathrm{N}$ stretching, $\mathrm{C}=\mathrm{C}$ stretching and stretching of sulfonyl group respectively. The molecular formula $\mathrm{C}_{20} \mathrm{H}_{19} \mathrm{~N}_{3} \mathrm{O}_{6} \mathrm{~S}_{2}$ was established by HR-MS showing molecular ion $[\mathrm{M}]^{+}$peak at $\mathrm{m} / \mathrm{z} 461.516$ (cald for $\mathrm{C}_{20} \mathrm{H}_{19} \mathrm{~N}_{3} \mathrm{O}_{6} \mathrm{~S}_{2}$ 461.513). In the EI-MS spectrum, four distinct peaks appeared at $\mathrm{m} / \mathrm{z}$ $149,147,121$ and 86 which pointed out the presence of 1,3-benzodioxol-5-yl carbonyl cation, 1,3-benzodioxol-5-yl cyanide cation, 1,3benzodioxole cation and morpholine cation fragments respectively in the molecule.

Table 2: Inhibition (\%) of selected bacterial strains by five 5-substituted-1,3,4-Oxadiazole-2-yl 4-(morpholin-4ylsulfonyl)benzyl sulfides (6a-e)

\begin{tabular}{ccccccc}
\hline \multirow{2}{*}{ Compound } & S. typhi (-) & E.coli (-) & K.pneumonae (-) & P.aeruginosa (-) & B. subtilis (+) & S. aureus (+) \\
\cline { 2 - 7 } & 6a & - & - & $75.35 \pm 0.06$ & $60.36 \pm 1.31$ & $54.55 \pm 2.95$ \\
6b & $75.00 \pm 0.33$ & $56.91 \pm 4.12$ & $55.59 \pm 1.14$ & $84.59 \pm 0.71$ & $82.20 \pm 0.54$ & $77.85 \pm 3.45$ \\
6c & $50.94 \pm 0.39$ & - & - & $76.94 \pm 0.12$ & $61.37 \pm 0.42$ & $61.70 \pm 0.80$ \\
6d & - & - & - & $59.71 \pm 3.03$ & $50.24 \pm 1.43$ & $51.75 \pm 1.65$ \\
6e & $55.22 \pm 1.78$ & - & $53.59 \pm 1.14$ & $50.32 \pm 2.08$ & $60.50 \pm 3.40$ & $57.06 \pm 0.12$ \\
\hline Ciprofloxacin & $\mathbf{9 1 . 1 4 \pm 0 . 8 4}$ & $\mathbf{8 8 . 9 4 \pm 1 . 2 1}$ & $\mathbf{9 0 . 6 5 \pm 1 . 2 9}$ & $\mathbf{9 0 . 3 6 \pm 1 . 8 6}$ & $\mathbf{9 1 . 3 3 \pm 1 . 9 9}$ & $\mathbf{9 1 . 8 7 \pm 2 . 5 1}$ \\
\hline
\end{tabular}


Table 3: MIC of five 5-substituted-1,3,4-Oxadiazole-2-yl 4-(morpholin-4-ylsulfonyl)benzyl sulfides (6a-e) against selected bacterial strains

\begin{tabular}{ccccccc}
\hline & \multicolumn{5}{c}{ MIC $(\mu \mathrm{M})$} \\
\cline { 2 - 7 } Compound & S. typhi (-) & E. coli (-) & K. pneumonae(-) & $\boldsymbol{P}$. aeroginosa (-) & B. subtilis (+) & S. aureus (+) \\
\hline $\mathbf{6 a}$ & $16.34 \pm 3.00$ & - & - & $12.39 \pm 1.45$ & $12.52 \pm 0.10$ & $17.28 \pm 4.07$ \\
$\mathbf{6 b}$ & $11.01 \pm 0.31$ & $15.37 \pm 3.33$ & $16.11 \pm 1.14$ & $9.70 \pm 1.96$ & $10.01 \pm 2.70$ & $9.15 \pm 0.29$ \\
$\mathbf{6 c}$ & $18.49 \pm 0.38$ & - & - & $13.16 \pm 3.45$ & $12.50 \pm 2.40$ & $13.99 \pm 0.86$ \\
$\mathbf{6 d}$ & - & - & - & $16.79 \pm 4.55$ & $19.87 \pm 2.20$ & $17.16 \pm 0.57$ \\
$\mathbf{6 e}$ & $16.56 \pm 2.23$ & - & $16.27 \pm 1.14$ & $18.86 \pm 2.10$ & $11.49 \pm 1.64$ & $17.58 \pm 3.21$ \\
\hline Ciprofloxacin & $\mathbf{8 . 2 6} \pm \mathbf{0 . 9 2}$ & $\mathbf{9 . 1 4} \pm \mathbf{0 . 2 8}$ & $\mathbf{8 . 9 2} \pm \mathbf{0 . 4 1}$ & $\mathbf{8 . 5 6 \pm 1 . 1 8}$ & $\mathbf{9 . 1 1} \pm \mathbf{1 . 3 0}$ & $\mathbf{8 . 4 8} \pm \mathbf{1 . 4 3}$ \\
\hline
\end{tabular}

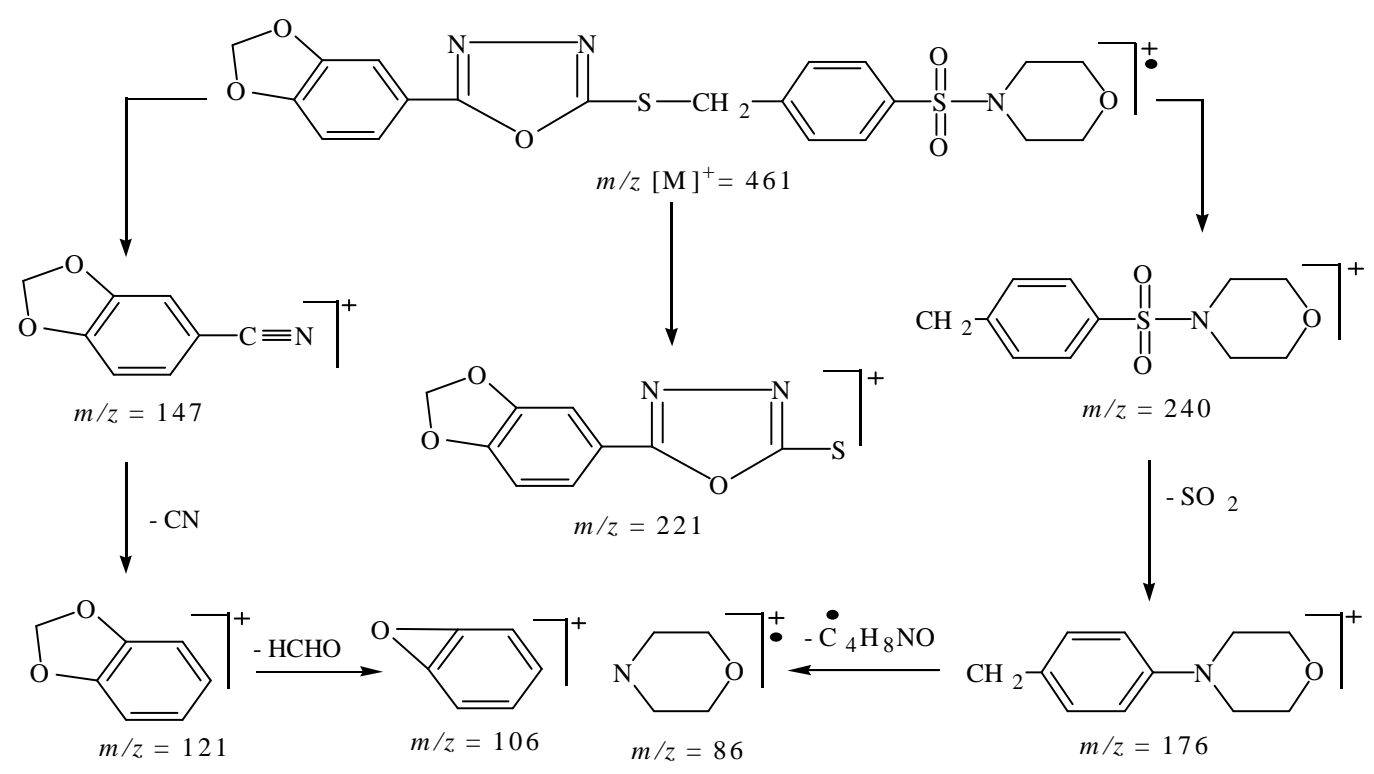

Figure 1: Mass fragmentation pattern of 5-(1,3-benzodioxol-5-yl)-1,3,4-oxadiazol-2yl-4-(morpholin-4yl sulfonyl)benzyl sulfide (6a)

The ${ }^{1} \mathrm{H}-\mathrm{NMR}$ spectrum showed four signals at $\delta$ $7.34\left(\mathrm{~d}, \mathrm{~J}=8.0 \mathrm{~Hz}, 1 \mathrm{H}, \mathrm{H}-6^{\prime}\right), 7.24(\mathrm{~d}, \mathrm{~J}=2.0 \mathrm{~Hz}$, $\left.1 \mathrm{H}, \mathrm{H}-4^{\prime}\right), 6.76\left(\mathrm{~d}, \mathrm{~J}=8.4 \mathrm{~Hz}, 1 \mathrm{H}, \mathrm{H}-7^{\prime}\right)$ and 5.89 (s, $2 \mathrm{H}, \mathrm{CH}_{2}-2^{\prime}$ ) in the downfield region owing to protons of 1,3-benzodioxole moiety attached to the 5-position of oxadiazole ring.

Two signals were resonated at $\delta 7.56(\mathrm{~d}, \mathrm{~J}=8.0$ $\mathrm{Hz}, 2 \mathrm{H}, \mathrm{H}-2$ " \& H-6") and 7.53 (d, J = 8.4 Hz, 2H, $\mathrm{H}-3$ " \& H-5"), due to higher coupling constant and each signal with integration of two protons, indicating the presence of para substituted aromatic ring. The two signals emerging at $\delta$ $9.54(\mathrm{~s}, 1 \mathrm{H}, \mathrm{CON}-\mathrm{H})$ and $4.36\left(\mathrm{~s}, 2 \mathrm{H}, \mathrm{CH}_{2}-2\right)$ which depicted the presence of acetamide group in the molecule. In the aliphatic region of the spectrum, two triplet were appeared at $\delta 3.57(\mathrm{t}$, $\mathrm{J}=4.4 \mathrm{~Hz}, 4 \mathrm{H}, \mathrm{CH}_{2}-2$ '" \& $\mathrm{CH}_{2}-6$ "') and $2.81(\mathrm{t}, \mathrm{J}=$ 4.4 Hz, 4H, $\mathrm{CH}_{2}-3^{\prime \prime \prime} \& \mathrm{CH}_{2}-5$ "') each with integration of four protons, showed the presence of morpholine ring and a methylene signal was appeared at 4.38 (s, $\left.2 \mathrm{H}, \mathrm{CH}_{2}-7^{\prime \prime}\right)$.
The mass fragmentation pattern of $\mathbf{6 a}$ was also clearly sketched in Fig 1. All the above signals collectively established the structure of compound 6a and named as 5-(1,3-benzodioxol5-yl)-1,3,4-oxadiazol-2yl-4-(morpholin-4yl sulfonyl)benzyl sulfide. Similarly, the structures of all the other synthesized compounds were elucidated with the help of above mentioned analytical techniques.

\section{Antibacterial activity}

Some of the synthesized compounds were moderate inhibitors of three bacterial strains employed in this study. One of the compounds, $\mathbf{6 b}$, exhibited significant activity against all the bacterial strains i.e. S. typhi (-), E. coli (-), K. pneumoniae (-), $P$. aeruginosa (-), B. subtilis (+) and $S$. aureus $(+)$ with $\mathrm{MIC}(\mu \mathrm{M})$ values of 11.01 $\pm 0.31,15.37 \pm 3.33,16.11 \pm 1.14,9.70 \pm 1.96$ $10.01 \pm 2.70$ and $9.15 \pm 0.29$ respectively. This compound was found to be more active because of the presence of 1,3-benzodioxol-5-yl ethenyl 
group attached to the oxadiazole ring present in the molecule. Against Pseudomonas aeruginosa, the order of activity of the compounds were $6 b>6 c>6 a>6 d>6 e$ from the highest activity to lowest activity but lower antibacterial activity than the standard compound used in this assay. Overall the activity revealed by the synthesized compounds was good as supported by their MIC values. Such kind of compounds can further be exploited and their derivatives could be synthesized to acquire closer MIC values to the standard, ciprofloxacin.

\section{CONCLUSION}

New compounds (6a-e) with antibacterial activity have been synthesized. One of the compounds inhibited the growth of four Gram negative ( $S$. typhi, E. coli, K. pneumoniae, P. aeruginosa) and two Gram positive (B. subtilis and $S$. aureus) bacterial strains. Thus, the compounds are potential lead molecules in the search for potent agents for the treatment of bacterial infections.

\section{REFERENCES}

1. Omar FA, Mahfouz NM, Rahman MA. Design, synthesis and anti-inflammatory activity of some 1,3,4oxadiazole derivatives. Eur J Med Chem 1996; 31: 819-825.

2. Goswami BN, Kataky JCS, Baruash JN. Synthesis and antibacterial activity of 1-(2,4-dichlorobenzoyl)-4substituted thiosemicarbazide,1,2,4-triazoles and their methyl-derivatives. $J$ Heterocycl Chem 1984; 21 : 1225-1229.

3. Holla BS, Poojary KN, Kalluraya B, Gowda PV. 5substituted-1,3,4-oxadiazolin-2-thiones. Indian $J$ Heterocyl Chem 1996; 5: 273-276.

4. Hasan A, Thomas NF, Gapil S. Synthesis, Characterization and Antifungal Evaluation of 5Substituted-4-Amino-1,2,4-Triazole-3-Thioesters. Molecules 2011; 16(2): 1297-1309.

5. Omar MT. Synthesis of new xanthenone derivatives of expected antibilharzial activity. Arch Pharm Res 1997; 20: 602-609.

6. Matsumoto $K$, Kawamura $Y$, Yasuda $Y$, Tanimoto $T$, Matsumoto $K$, Yoshida $T$, Shoji J. Isolation and characterization of thioxamycin. $J$ Antibiot (Tokyo) 1998; 42: 1465-1469.

7. Shafi SS, Radhakrishnan TR. Synthesis and antibacterial activity of some 2,5-di-substituted-1,3,4-oxadiazole, 1,3,4-thiadiazole, 1,2,4-triazole and 4-thiazolidinone. Indian J Heterocyl Chem 1995; 5: 133-138.

8. Tan TM, Chen Y, Kong KH, Bai J, Li Y, Lim SG, Ang H, Lam $Y$. Synthesis and the biological evaluation of 2benzenesulfonylalkyl-5-substituted-sulfanyl-(1,3,4)oxadiazoles as potential anti-hepatitis $B$ virus agents. Antiviriral Res 2006; 71: 7-14.

9. Wagle S, Vasudeva AA, Suchetha NK. Synthesis of some new 2-(3-Methyl-7-substituted-2-oxoquinoxalinyl)-5-(aryl)-1,3,4-oxadiazoles as potential nonsteroidal anti-inflammatory and analgesic agents. Indian J Chem 2008; 47B: 439-448.

10. Padmavath V, Reddy GS, Padmaja A, Kondaiah $P$, Shazia A. Synthesis, antimicrobial and cytotoxic activities of 1,3,4-oxadiazoles, 1,3,4-thiadiazoles ann 1,2,4-triazoles. Eur J Med Chem 2009; 44: 21062112.

11. Hanif M, Hussain M, Ali S, Bhatti MH, Ahmed MS, Mirza $B$, Stoeckli-Evans $H$. Biological studies and structural elucidation of Organotin(IV) derivatives of 6nitropiperonylic acid: Crystal Structure of $\{[(\mathrm{CHOCH}(\mathrm{O}-\mathrm{NO}) \mathrm{COO}) \mathrm{SnBu} 2 \mathrm{2O}\}$. Polyhedron 2010; 29(1): 613-619.

12. Zou X, Zhang Z, Jin G. Synthesis and biological activity of 1,3,4-oxadiazole-substituted pyridazinones, J. Chem. Res. Synopses 2002; 5: 228-230.

13. Aziz-ur-Rehman, Fatima A, Abbas N, Abbasi MA, Khan KM, Ashraf M, Ahmad I and Ejaz SA. Synthesis, characterization and biological screening of 5substituted-1,3,4-oxadiazole-2yl-N-(2-methoxy-5chlorophenyl)-2-sulfanyl acetamide. Pak J Pharm Sci 2013; 26(2): 345-352.

14. Aziz-ur-Rehman, Fatima A, Abbasi MA, Rasool S, Malik A, Ashraf M, Ahmad $I$ and Ejaz SA (2013b). Synthesis of new N-(5-chloro-2-methoxyphenyl)-4-(5substituted-1,3,4-Oxadiazol-2-ylthio)butanamide derivatives as suitable lipoxygenase inhibitors. J Saudi Chem Soc 2013; doi: http://dx.doi.org/ 10.1016/j.jscs.2013.02.006.

15. Khalid H, Aziz-ur-Rehman, Abbasi MA, Malik A, Rasool S, Nafeesa K, Ahmad I and Afzal S. Synthesis, spectral analysis and anti-bacterial study of $\mathrm{N}$ substituted derivatives of 2-(5-(1-(phenylsulfonyl) piperidin-4-yl)-1,3,4-Oxadiazol-2-ylthio)acetamide. $J$ Saudi Chem Soc 2013; doi: http://dx.doi.org/ 10.1016/j.jscs.2013.05.001.

16. Kaspady M, Narayanaswamy VK, Raju M, Rao GK. Synthesis, antibacterial activity of 2,4-disubstituted oxazoles and thiazoles as bioesters. Lett Drug Des Discov 2009; 6: 21-28.

17. Yang CR, Zang Y, Jacob MR, Khan SI, Zhang YJ, Li XC. Antifungal activity of $\mathrm{C}-27$ steroidal saponins. Antimicrob Agents Ch 2006; 50(5): 1710-1714. 